\title{
Optimization of the process conditions for minimizing the deactivation in the furfural-cyclopentanone aldol condensation in a continuous reactor
}

\author{
Jennifer Cueto, Laura Faba, Eva Díaz, Salvador Ordóñez \\ Catalysis, Reactors and Control Research Group (CRC), Dept. of Chemical and Environmental \\ Engineering, University of Oviedo, Oviedo 33006, Spain \\ *e-mail: sordonez@uniovi.es, Tel: +34 985103 437; Fax: + 34985103434
}

\begin{abstract}
This work demonstrates the key role of salts (both anions and alkaline cations) in the activity and stability of Mg-Zr mixed oxide as catalyst for the furfural-cyclopentanone aldol condensation in a continuous fixed bed reactor. Reactants oligomers are shown to cause active sites blockage, controlling the reaction conditions being required to maximize the selectivity for the first condensation adduct.
\end{abstract}

The best results were obtained at $\mathrm{pH}=8$, using $\mathrm{KHCO}_{3}$ for fixing this $\mathrm{pH}$. At these conditions, 75 $\%$ and $14 \%$ of $\mathrm{C} 10$ and $\mathrm{C} 15$ yields were observed at $8 \mathrm{~h}$ on stream, whereas experiments performed at the other conditions show almost total deactivation. This good performance (performance and stability) is explained by a synergistic effect of two phenomena: a good buffering activity of the anion, and the ion exchange involving $\mathrm{Na}$ and $\mathrm{K}$, which leads to the stabilization of the basic sites.

Keywords: Continuous fixed bed reactor; MgZr mixed oxide; deactivation mechanism; K-Mg exchange; base-catalyzed reactions; biomass catalytic upgrading 


\section{Introduction}

The growing concern about global climate change has encouraged the development of renewable alternatives to obtain fuels and chemicals, introducing the "bio-refinery" concept and promoting the use of biomass as carbon source in chemical processes $[1,2]$. In this context, the transformation of residual lignocellulosic biomass into biofuels by aqueous-phase reactions is one of the most promising strategies [3-6], highlighting the four-step route firstly proposed by Dumesic and co-workers [7]. The aldol condensation of furfural (FFL) using a ketone as linking molecule is the key step of this process. Thus, it has attracted attention of the scientific community in the last few years, focusing the efforts on the development of active and selective homogeneous ( $\left.\mathrm{NaOH}, \mathrm{Ca}(\mathrm{OH})_{2}\right)$ and heterogeneous ( $\mathrm{Mg}-\mathrm{Zr}$ or Mg-Al mixed oxides) catalysts [8-14].

All these previous studies were performed in a batch (discontinuous) reactor, analyzing the influence of main parameters in the conversion and selectivity to desired products. However, the research in continuous systems allows determining the stability of these catalysts, an essential parameter for their implementation at industrial scale. Despite these facts, there are only few studies about the furfural and acetone cross condensation deactivation in batch reactors $[15,16]$ and only one reference studied the stability of this reaction in fixed bed configuration [17]. Main conclusion of this research is related to the instability of hydrotalcites at reaction conditions, with a fast decrease in furfural conversion as well as in the selectivity to the main aldol condensation products. This deactivation is justified by the presence of strong basic sites that catalyze side reactions related to self-condensations of both reactants with concomitant oligomerization reactions, resulting into very complex deactivation mechanisms. This activity loss, caused by deposition of organic oligomers, has been also described in other aldol condensations of biomass derivatives, both in liquid and gas phase and despite the catalyst used, highlighting the high relevance of this phenomenon as well as the interest of reducing it [18-20]. The low solubility of the condensation adducts in water promotes catalyst 
deactivation by fouling. Although the cationic species of these solids are slightly soluble in water, there is not any study about the effect of this fact on catalyst stability.

These undesired reactions could be prevented or minimized if the main reaction is promoted, reducing the self-condensation capacity. With this aim, we have studied the acetone substitution by other renewable ketone: cyclopentanone (CPO) [21]. CPO is considered as a very interesting bioplatform molecule, obtained from furfural via rearranging reaction [22-26]. In addition, CPO has five carbon atoms, yielding larger ketone-furfural adducts (C10 - C15). These advantages have promoted the development of several works proposing the furfuralCPO system as a good alternative for obtaining long-chain carbon products $[21,27-30]$. As we have discussed in our previous work, CPO self-condensation kinetic is significantly slower than the acetone's one, whereas the cross-condensation with furfural is strongly activated (kinetics 300 times faster than with acetone), obtaining complete conversion and higher selectivities, even working at milder conditions (overall yield to the products of $77 \%$ after $4 \mathrm{~h}$ of reaction at $303 \mathrm{~K}$ ) [21]. However, as consequence of the large size of products, the main compounds are obtained as a solid phase.

Condensation adducts insolubility is a strong drawback for the study of this reaction in continuous flow. Different combinations of organic solvents with water in biphasic or binary systems were studied in a second approach of this reaction. An ethanol/water mixture (2:1 volume ratio) was found the optimum to reach the equilibrium between condensation kinetics (water is required in the limiting steps) and solubility (higher in organic phases), with almost $40 \%$ of overall yield after $24 \mathrm{~h}$ [31]. Once the insolubility was significantly reduced, its implementation in continuous flow can be approached, aim of the present study. Different aspects, as the reactant molar ratio, reaction medium $\mathrm{pH}$ or the role of the leaching/ion exchange were considered in this study. All the reaction results were complemented with catalyst characterization, in order to follow the evolution of the catalytic properties during the reactions, with the aim to explain the experimental results as function of lixiviation, adsorption 
and precipitation phenomena, proposing an alternative approach to this reaction with higher activity and selectivity.

\section{Experimental}

\subsection{Synthesis and characterization of fresh Mg-Zr mixed oxide}

The synthesis procedure of this mixed oxide is based on the solid-gel technology detailed in our previous works $[14,21]$. Both nitrates, $\mathrm{Mg}\left(\mathrm{NO}_{3}\right)_{2} \cdot 6 \mathrm{H}_{2} \mathrm{O}$ (Sigma Aldrich, $98 \%$ ), $\mathrm{ZrO}\left(\mathrm{NO}_{3}\right)_{2}$ (Sigma Aldrich, $99 \%$ ) were dissolved in water, producing the precipitation of the mixed oxide precursor at $\mathrm{pH} 10(\mathrm{NaOH}$ is used as precipitating agent). After $24 \mathrm{~h}$ aging at $353 \mathrm{~K}$, the whitish solid was filtered and washed to $\mathrm{pH} 7$. Thus, it was dried at $373 \mathrm{~K}$ and treated in a He flow with a temperature increase to $873 \mathrm{~K}\left(5 \mathrm{~K} \cdot \mathrm{min}^{-1}\right)$. Temperature was held for $3 \mathrm{~h}$.

Mg-Zr mixed oxide catalyst (henceforth, Mg-Zr) was characterized using different techniques in order to study the main surface, morphological and physic-chemical properties relevant to its catalytic activity. All the characterization results, as well as the methodology carried out, are deeply explained in our previous works $[14,21,31]$. These properties are mentioned and referred when they are required for justifying the experimental results. Isoelectric point was measured in a Zetasizer Nano ZS Instrument (Malvern Instruments Ltd., UK) with the solution of $\mathrm{CPO}$ and $\mathrm{FFL}$ in water and ethanol at different $\mathrm{pH}$.

\subsection{Catalytic studies}

The reaction was conducted in a stainless-steal fixed-bed reactor with $1 / 2$ " of internal diameter and $6 \mathrm{~cm}$ length, submerged in a water bath equipped with a temperature control. The feed was also preheated to the operation temperature ( $323 \mathrm{~K})$ before being pumped into the system, using an electric heater with a magnetic stirrer in order to ensure homogeneous properties. 
The catalyst bed is configured with the catalyst, $0.5 \mathrm{~g}$ of $\mathrm{Mg}-\operatorname{Zr}(250-355 \mu \mathrm{m})$, located between two inert zones of $2.25 \mathrm{~cm}$ in length packed with glass balls $(1 \mathrm{~mm})$ for preventing preferential channels and dead volumes. These parameters were chosen to ensure that reaction takes place under ideal packed bed conditions [32]: ratio between reactor length and particle size higher than 100 (240 at working conditions); ratio between reactor diameter and particle size higher than 10 (50.8 in this case). A continuous flow of $1 \mathrm{~mL} \cdot \mathrm{min}^{-1}$ is fed into the system using a HPLC pump, containing a $5 \%$ mass concentration of furfural (Sigma Aldrich, $99 \%$ ) and cyclopentanone (Sigma Aldrich, $99 \%$ ) dissolved in a mixture of ethanol/water (2:1 volume ratio). The feed stream was heated before reaching the catalyst.

The reaction evolution was analyzed periodically by gas chromatography, using a Shimadzu GC-2010 equipped with an FID detector (CP-Sil 8 CB of $30 \mathrm{~m}$ length as a stationary phase). The identification of the main products was verified with GC-MS (Shimadzu GC / MS QP2010 Plus), using a TRB-5MS capillary column (30 m length). The calibration of CPO, FFL and the first adduct obtained (labeled as C10) was carried out using commercial standards provided by Sigma Aldrich, while the second adduct (C15) signal was quantified based on the response factor method proposed by Scanlon and Willis [33]. Condensation adducts yields (as function of the limiting reactant) and carbon balance were calculated according to the following equations:

$$
\begin{gathered}
\Psi_{C 10} \%=\frac{m o l ~ C 10}{\mathrm{~mol} \mathrm{FFL} \mathrm{L}_{t=0}} \cdot 100 \\
\Psi_{C 15} \%=\frac{2 \cdot \mathrm{mol} \mathrm{C15}}{\mathrm{mol} \mathrm{FFL} L_{t=0}} \cdot 100 \\
\text { C.B. }(\%)=\frac{5 \cdot \mathrm{mol} \mathrm{CPO}+5 \cdot \mathrm{mol} \mathrm{FFL}+10 \cdot \mathrm{mol} \mathrm{C10}+15 \cdot \mathrm{mol} \mathrm{C} 5}{5 \cdot \mathrm{mol} \mathrm{CPO}_{t=0}+5 \cdot \mathrm{mol} \mathrm{FFL} t=0} \cdot 100
\end{gathered}
$$

The presence of internal and external mass-transfer limitations was checked by WheelerWeisz criteria and Carberry number [34] at the initial reaction times, for the most active 
catalyst and reaction conditions observed in this work. Internal and external mass-transfer contributions values of $1.8 \cdot 10^{-2}$ and $1.2 \cdot 10^{-4}$, respectively, discard these limitations, since the limit to be considered are 0.1 for the internal (Wheeler-Weisz criteria) and 0.05 for the external one (Carberry number) [34]. These studies are detailed in the Supplementary Information.

\subsection{Characterization of used catalysts}

To identify any modification of the catalyst surface during the reaction, catalysts were recovered after each reaction, dried overnight at $373 \mathrm{~K}$ and analyzed by different techniques. Morphological change on the Mg-Zr surface was checked by scanning electron microscopy (SEM), performed with a JEOL-6610LV SEM. TG-DSC instrument (Setaram, Sensys) was used treating the samples $(20 \mathrm{mg})$ in a nitrogen flow $\left(20 \mathrm{~mL} \cdot \mathrm{min}^{-1}\right)$ from 298 to $973 \mathrm{~K}$ at $5 \mathrm{~K} \cdot \mathrm{min}^{-1}$. The surface composition was measured by X-ray Photoelectron Spectroscopy (XPS) using a SPECS system equipped with a Hemispherical Phoibos detector.

Carbonaceous deposits were studied by TPO (temperature-programmed oxidation) after the appropriate mechanical homogenization, using a Micromeritics TPD/TPR 2900. Samples were treated in oxygen flow $\left(2 \% \mathrm{O}_{2}\right.$ in $\left.\mathrm{He}\right)$, increasing the temperature up to $1273 \mathrm{~K}$ at a $2.5 \mathrm{~K} \cdot \mathrm{min}^{-1}$ rate. The evolution of $\mathrm{CO}_{2}$ signal was measured with a mass spectrometer Pfeiffer Vacuum Omnistar Quadrupole Mass Spectrometer. Similar procedure, but under inert atmosphere (helium flow), was carried out to determine the possible adsorption of reactants and products. The identification of the analyzed compounds was corroborated by comparison to the theoretical $\mathrm{m} / \mathrm{z}$ spectra data compiled by NIST Mass Spec Data Center [FFL $(\mathrm{m} / \mathrm{z}=96,95,39$, $38,29,67) ; \mathrm{CPO}(\mathrm{m} / \mathrm{z}=84,55,28,84,41,56) ; \mathrm{C} 10(162,120,106,91,78,55,39) ;$ and $\mathrm{C} 15$ $(240,106,91,78,81,55,39)]$. Signals of $m / z=39$ and 55 were chosen to quantify FFL and CPO units in reactants and products. 


\section{Results and discussion}

\subsection{Preliminary test based on optimized batch conditions}

A first approach to continuous mode was carried out at $323 \mathrm{~K}$, using a binary solvent system ethanol/water (2:1 volume ratio) and an equimolar CPO:FFL feed with a global $5 \%$ wt.

concentration $\left(\right.$ WHSV $\left.=4.6 \mathrm{~h}^{-1}\right)$ [31]. Figure 1 shows the conversion evolution and the products profiles as function of time on stream (TOS), these deactivation effects being notorious. Thus, after $8 \mathrm{~h}, \mathrm{CPO}$ and FFL conversions decrease from $45 \%$ and $38 \%$, to 15 and $5.2 \%$, respectively. The main product, $\mathrm{C} 10$ reaches a maximum concentration of $35 \mathrm{mmol} \cdot \mathrm{L}^{-1}$ at low TOS, corresponding to a yield of $12.6 \%$, followed by a continuous decrease until disappearance after $4 \mathrm{~h}$. By contrast, C15 is hardly detected in quantitative amounts during the first hour ( $2 \mathrm{mmol} \cdot \mathrm{L}^{-1}$ as maximum concentration achieved, right axis in Fig. $1 \mathrm{~b}$ ), with a total disappearance at higher TOS.

CPO self-condensation is discarded, since no CPO dimers were detected in the liquid phase, and the theoretical solubility of CPO dimmer (estimated by empirical correlations proposed by Stein and Brown [35]) is four times higher than the solubility of $\mathrm{C} 10$ adduct $\left(0.046 \mathrm{~mol} \cdot \mathrm{L}^{-1} \mathrm{vs}\right.$. $\left.0.01 \mathrm{~mol} \cdot \mathrm{L}^{-1}\right)$. Furthermore, $\mathrm{CPO}$ self-condensation takes place on the same active sites as the FFL-CPO cross-condensation (medium-strength basic sites) [36]. Thus, at these conditions cross-condensation predominates over the CPO self-condensation [21]. 


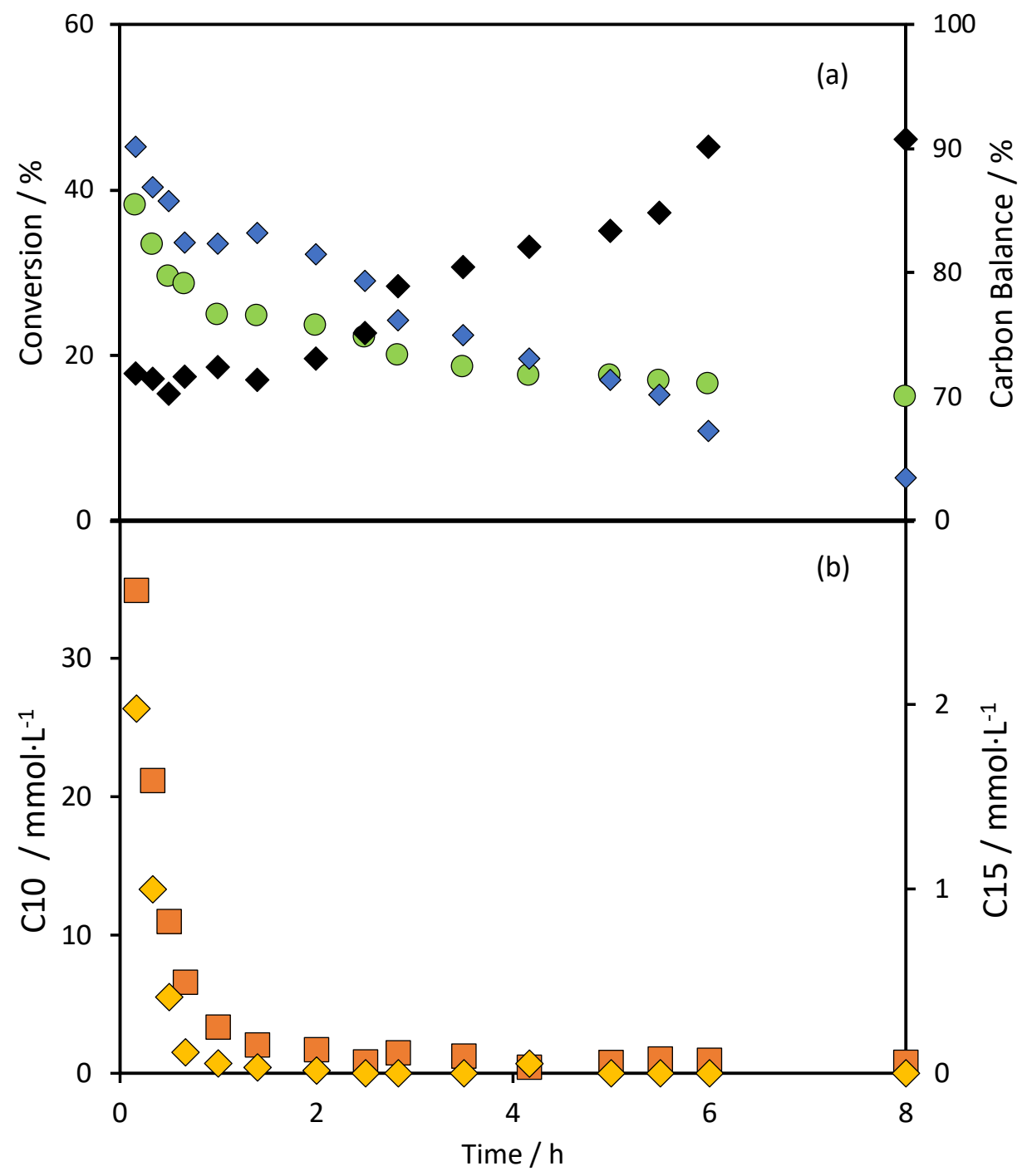

Figure 1. Experimental results obtained in the reaction carried out at WHSV $=4.6 \mathrm{~h}^{-1}, 323 \mathrm{~K}, 1: 1 \mathrm{CPO}: \mathrm{FFL}$ molar ratio, 2:1 EtOH/water; $\mathrm{pH}$ 4.75. (a) Conversion evolution of FFL $(\bullet)$ and $\mathrm{CPO}(\bullet)$ as well as carbon balance $(\bullet)$. (b) Concentration of reaction products: C10 $(\boldsymbol{\square})$, C15 $(\diamond)$.

There is not agreement between reactants conversions and the corresponding concentrations of both products (these values would correspond to theoretical conversions of 14 and $13.3 \%$, of FFL and CPO, respectively). Consequently, the carbon balance closure (Fig. 1a) is quite low at the initial TOS (72\%), increasing after to values higher than $90 \%$. A possible cause of these discrepancies could be the reaction between furfural and ethanol (producing the 
corresponding acetal), previously reported mainly with acid catalysis [37]. However, experimental analyses indicate that less than $1 \%$ of furfural is converted to the acetal, due to the different kinetics of both reactions, condensation and acetylation.

Once the acetylation side reaction is discarded, the inconsistences between furfural conversion and carbon balance closure can be explained by two concomitant reasons: (1) a strong adsorption of reactants on the catalytic surface; and/or (2) side reactions yielding solid products. Significant adsorption at the initial times cannot be discarded, according to individual adsorption tests of each component (Fig. S1). It must be pointed out that no liquid products are detected when FFL or CPO are fed individually, suggesting that, if any selfcondensation is taking place, the products obtained are solids. As the CPO self-condensation products are soluble in water [38], this self-condensation is discarded. These profiles, in addition to adsorption of both compounds, suggest a relevant FFL oligomerization, result justified by the long time required to obtain the stable FFL signal. In good agreement with this hypothesis, CPO signal was obtained after washing the catalyst saturated in this compound, whereas FFL was not desorbed after an analogous procedure. It can be concluded that two different adsorption phenomena are taking place: CPO physisorption and chemisorption with oligomerization of FFL, obtaining a stable solid in the second case.

According to these adsorption studies, FFL oligomerization has been proposed as the main cause of this deactivation. This hypothesis is congruent with the remaining FFL conversion despite the low conversion of CPO as well as the absence of any product in the liquid phase, Fig 1a. However, a deeper analysis is required to identify why this deactivation is so evident at continuous mode, whereas it was not observed under batch conditions [21]. Spent catalyst was recovered after $8 \mathrm{~h}$ and analyzed by different techniques. Morphological analysis by SEM microscopy demonstrates an evolution of the surface, with the appearance of scale structures covering the amorphous initial surface (Figure 2). 

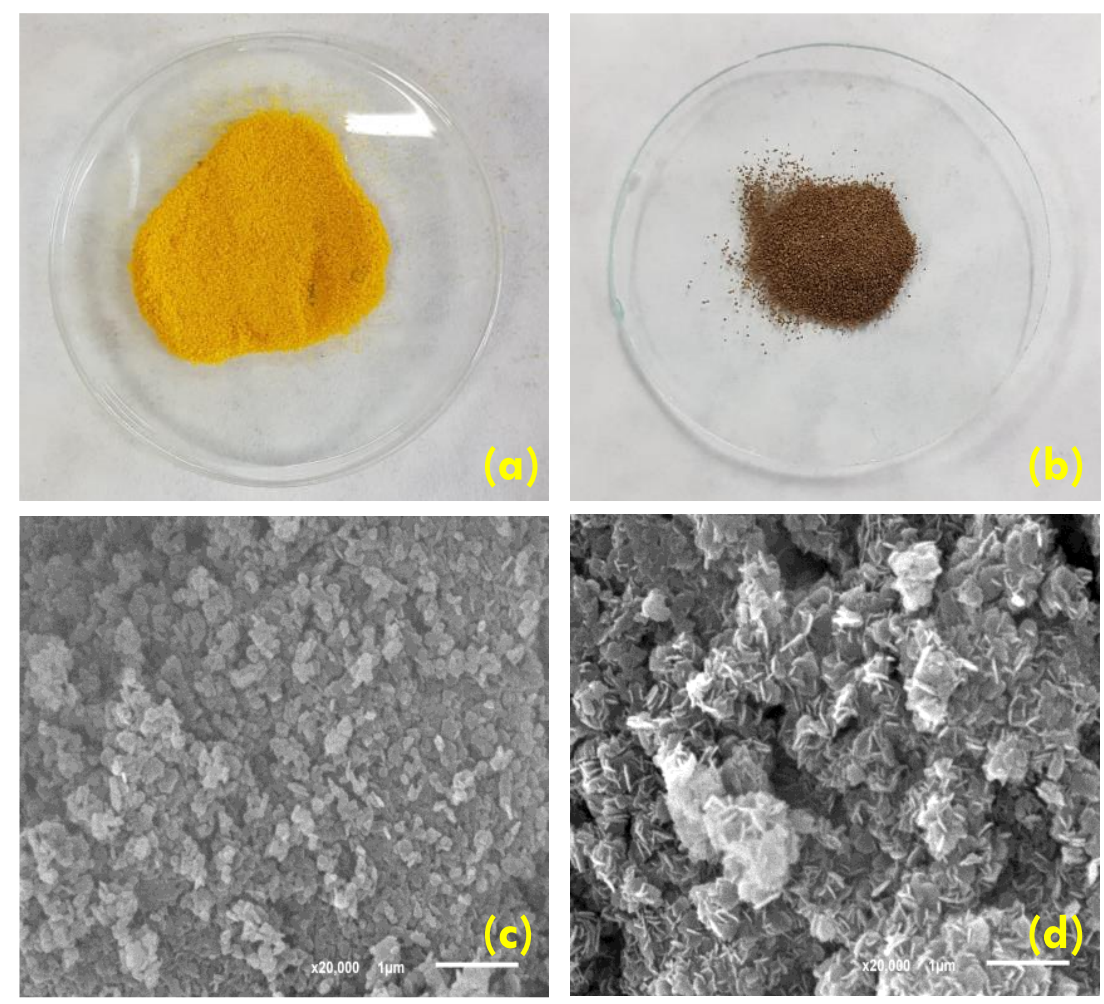

Figure 2. Macroscopic aspect of solids recovered after reaction under (a) batch and (b) continuous flow; Scanning-electron microscopy (SEM) micrographs of (c) fresh Mg-Zr and (d) recovered Mg-Zr after reaction.

These structures suggest the presence of solid deposits, being different from those obtained after the batch experiments. These results were expected, considering the qualitative analysis of the macroscopic appearance (crystalline aspect in the batch conditions but a dark and amorphous surface after the reaction in continuous flow). The presence of carbonaceous deposits was corroborated by XPS analyses. The study of the spent catalyst indicates a Mg-Zr surface with almost $24 \%$ of carbon (being the Mg-Zr fresh catalyst free of this element). The $\mathrm{Mg} / \mathrm{O}$ and $\mathrm{Zr} / \mathrm{O}$ ratio remains constant after the reaction. This fact, as well as the ICP results, discards a relevant role of metal leaching (50 and $0.007 \mathrm{ppm}$ of $\mathrm{Mg}$ and $\mathrm{Zr}$ detected in the reaction liquor). Thus, the permanent deposition of organic compounds is proposed as the main deactivation cause. 
The spent catalyst was suspended in a solution of chloroform, for solving and identifying the organic compounds. The analysis of the chloroform phase after the leaching test only shows a small signal of C15 product and other compound with a complex carbon structure, including aromatic rings. It must be pointed out that the catalyst after the leaching test is still dark, a clear consequence of stables compounds not dissolved. Similar analysis after the batch experiment demonstrates the presence of only $\mathrm{C} 10$ and $\mathrm{C} 15$ compounds, recovering the white color after the extraction [21].

These results are congruent with the evolution of signals obtained with the temperatureprogrammed analyses under inert atmosphere (TPD). In order to guarantee a complete identification of spectra obtained, these results were compared with those obtained after studying the desorption of each compound as well as the TPD of spent catalysts obtained at batch conditions (well identified products). The discussion of these results is detailed in the supplementary information, including the corresponding TPD spectra (Figure S2).

During the TPD analysis, the $\mathrm{m} / \mathrm{z}$ fragment of 55 allows following CPO release, whereas 39 is used for FFL. These fragments, and not those corresponding to their molecular weights, were chosen because of different reasons: (1) calibration limitations of the instrument (higher sensitivity to masses lower than 74); (2) the expected partial fragmentation of large molecules under analysis conditions; (3) the assignation is unequivocal to each reactant; (4) these signals (39 and 55) are present in the decomposition of both reaction products. This last point was checked with the mass spectra of each compound obtained by GC-MS (Figure S3). Based on these conclusions, the second peak obtained in the TPD of continuous-reaction sample $(723 \mathrm{~K})$ indicates the presence of $\mathrm{C} 10$ adsorbed, whereas the first one $(\approx 600 \mathrm{~K})$ can be deconvoluted into two signals, being related to C15 (613 K) and furfural (594 K). The slight displacement of this signal respect to the signal obtained with the pure compound is congruent with a partial oligomerization of this compound. This last peak is the main contribution and it was not observed in batch conditions. 
The strength of this adsorption effects is not enough to explain the catalytic deactivation, since the catalyst after the TPD is still dark, whereas the catalyst of the discontinuous reaction recovers the original white color. This fact is congruent with the thermogravimetric analyses (Fig. S4), with a mass loss lower than $30 \%$ at continuous mode, significantly lower than the $80 \%$ of the sample after batch reaction. Considering the remaining oligomers are stable up to $1273 \mathrm{~K}$ under inert conditions, a TPO analysis (oxidizing atmosphere) of sample after TPD was carried out (Fig. S4). A complex profile is obtained, consequence of the oxidation of different compounds, being congruent with the hypothesis previously proposed: different oligomers adsorbed on the catalytic surface. As it could be expected, a similar analysis of spent catalysis after batch reaction does not give any signal (all the solids were removed by the previous desorption in the TPD experiment).

The different behavior between batch and continuous flow can be associated to the different contact between the organics and the catalyst. Whereas under batch conditions the catalyst is suspended in the bulk solution, the plug-flow configuration at these working conditions implies a significant increase in the contact time. This fact justifies a higher relevance of adsorption processes. In the same way, final products desorption is also slower, promoting oligomerization routes. As it was explained in the experimental section, and detailed in the supplementary information, the mass transfer control has been discarded.

According to this discussion, efforts to prevent or minimize this deactivation must be firstly focused on tuning the initial reactant ratios to maximize the $\mathrm{C} 10$ production, reducing the $\mathrm{C} 15$ one, and also these side-reactions related to an excess of FFL. Thus, a screening of different initial reactant ratios was carried out in order to identify the optimal conditions for this flow regime.

\subsection{Reactant ratio (CPO:FFL) in the feed}

Different experiments were performed keeping constant the total molar concentration of organics and the reaction conditions ( 5 wt. $\%, 4.6 \mathrm{~h}^{-1}, 323 \mathrm{~K}$ and $\left.\mathrm{pH} 4.75\right)$, but with increasing 
CPO:FFL ratios (5:1, 10:1 and 15:1). An extra experiment with FFL excess (1:10) was also carried out without obtaining any significant conversion, even at low TOS. Main results of different CPO excess studied are shown in Figure 3, whereas the complete evolution with the time is shown and deeply analyzed in the supplementary information (Fig. S5).
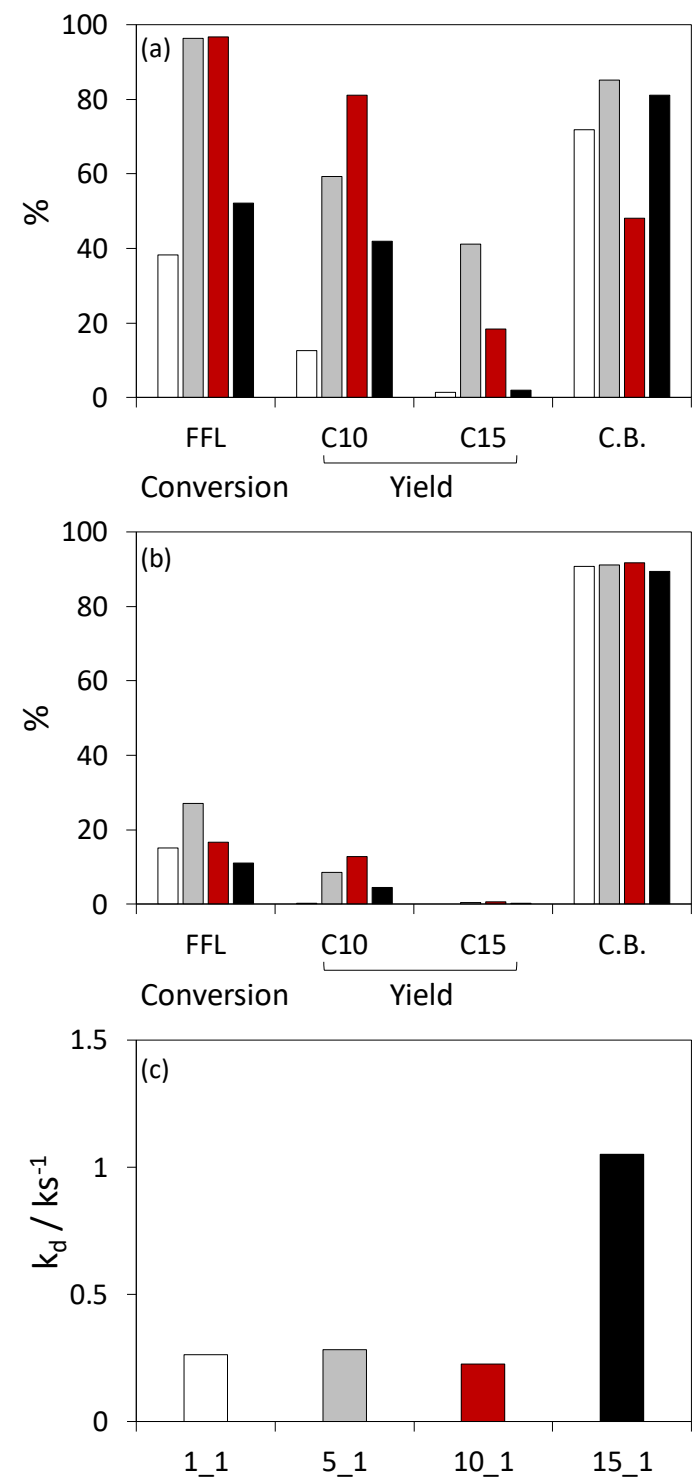

Figure 3: Summary of main data obtained in the CPO and FFL condensation at $323 \mathrm{~K}$ as function of the initial CPO:FFL ratio. Values related to: (a) $t=0 ;(b) t=8 \mathrm{~h}$ (c) deactivation kinetic constant. Data correspond to 1:1 (white); 5:1 (grey); 10:1 (red); 15:1 (black).

For the same reason, the analysis of products yields is carried out based only on the limiting reactant (FFL), using the equation (2) detailed in the experimental section. The expected increase in the furfural conversion as the CPO:FFL ratio increases is observed (limiting 
reactant), except in the case of the 15:1 reaction. This suggests a poor adsorption of FFL in these conditions (competitive adsorption of CPO on the same types of sites), inhibiting the reaction. The highest activity at initial times is obtained with 5:1, with C10 and C15 yields of 59 and $43.5 \%$, respectively. At $10: 1$, there is not furfural enough to promote the $\mathrm{C} 15$ in so large amount (initial yield of $18.4 \%$ ), obtaining more than $80 \%$ of the first adduct (C10). Concerning the products evolution with the reaction time, the highest deactivations are observed at the extreme conditions. In the case of 15:1 reaction, this is congruent with the high value of the deactivation kinetic constant $\left(\mathrm{k}_{\mathrm{d}}=1.05 \mathrm{ks}^{-1}\right)$, whereas this parameter is not very high in the case of 1:1 $\left(\mathrm{k}_{d}=0.263 \mathrm{ks}^{-1}\right)$. This is congruent with a high relevance of furfural side-reactions at these conditions; consuming this compound despite no products are observed (fast deactivation due to furfural oligomerization). This hypothesis is supported by the temporal evolution of carbon balance (see supporting information), clearly different from the evolutions at other conditions. The remaining C10 observed at 5:1 and 15:1 indicates only a partial deactivation. At these cases, the loss of activity is the result of a combination of $\mathrm{C} 15$ stable adsorption and furfural oligomerization. This last cause is almost negligible in the case of $10: 1$, as it is concluded comparing the amount of furfural required to obtain the C10 and C15 and the amount really consumed (see the entire discussion in the supplementary information). These hypotheses are in good agreement with the evolution of the spent catalyst colors, shown in Figure S6, with an evolution from the dark brown aspect of catalyst obtained with 1:1 reaction, to a clearer yellow aspect at 10:1, being more congruent with the deposition of C15 compounds (as in the case of the batch conditions). The different color of the catalysts after the 15:1 experiment also suggests a different mechanism at these conditions. Considering the qualitative character of these macroscopic images, TPD analyses were carried out to quantify and validate these conclusions. Main profiles are shown in Figure 4. 


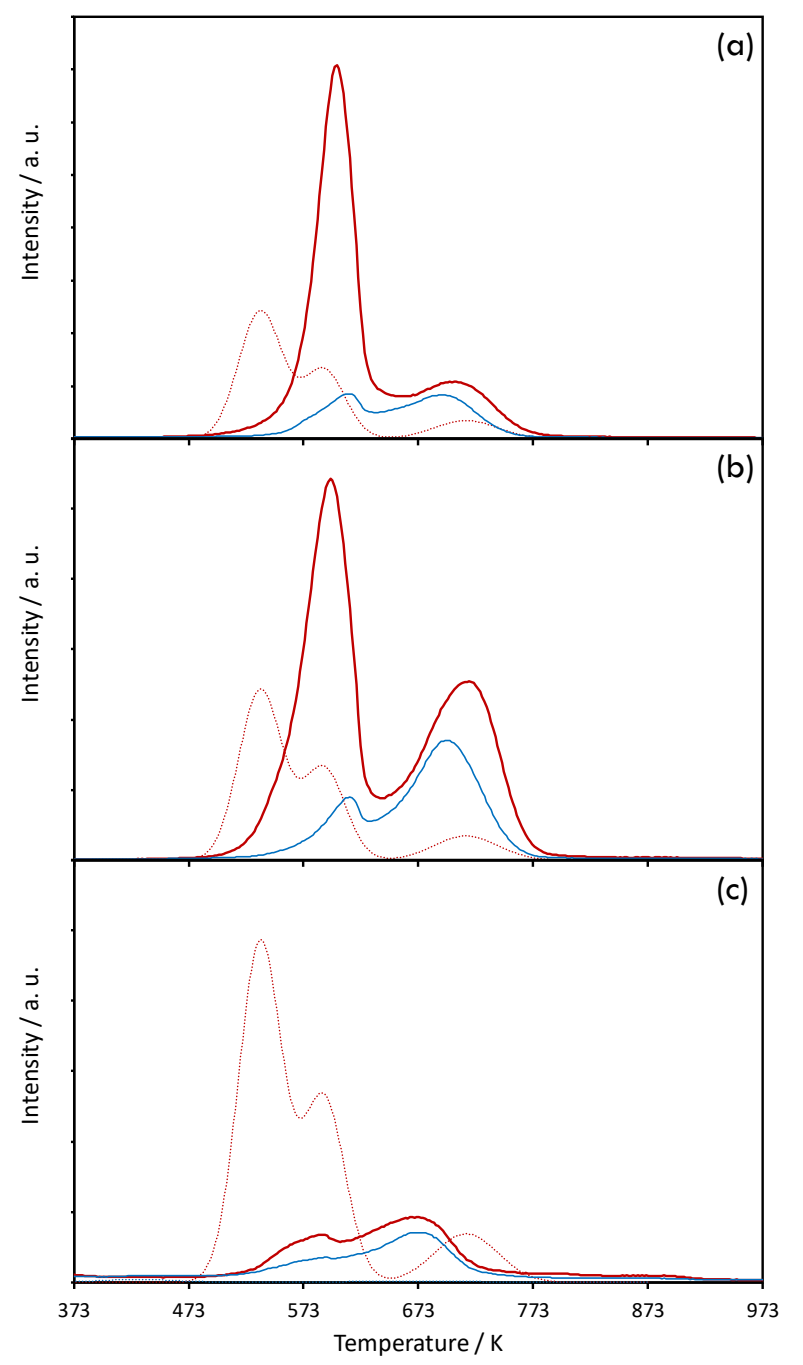

Figure 4: TPD analyses of spent catalysts recovered after reaction at different CPO:FFL ratios:

(a) $5: 1$; (b) $10: 1$; (c) 15:1. Blue lines correspond to CPO signals; whereas FFL ones are plotted in red color. Results of dotted lines correspond to individual saturations with each reactant.

In these profiles, the signals obtained after the desorption of catalysts saturated on individual reactant are also plotted, with the aim of identifying the desorption temperatures but also to compare the different scales used. It is must be pointed out that signal related to the CPO desorption is negligible at any of these scales. The aspect of the desorption profile catalyst working with the $15: 1$ is clearly different, whereas in the other cases, same peaks as for the 1:1 were obtained (but with different intensities). This new peak obtained at $15: 1$ appears at $673 \mathrm{~K}$ and involves both, FFL and CPO units. However, CPO signal is directly proportional to the FFL 
one, in opposition to the other results. In addition, the signal related to main product adsorption does not appear in the spectra at 15:1. Considering that heavy compounds are not detected by these analyses, the main conclusion is related to the higher signals obtained with 5:1 and 10:1 samples, in comparison to the previous ones obtained at 1:1. This suggests a higher control of the reaction in the range of $\mathrm{C} 15$ and $\mathrm{C} 10$ compounds, discarding a relevant amount of furfural-derived heavy products. Moreover, the relative relevance of C15 signals decreases as CPO:FFL ratio increases, being congruent with the proposed hypotheses of activity and deactivation. In good agreement with these assumptions, the oxidation peak of reaction 5:1 at $\approx 585 \mathrm{~K}$ (TPO analyses, included in Figure $\mathrm{S} 7$ ) is the highest one, suggesting a higher amount of $\mathrm{C} 15$ than at 1:1. The lowest amount of carbonaceous deposits on the catalyst corresponds to $10: 1$ conditions. Finally, the difference in the oxidation temperature of deposits obtained at 15:1 ( $\approx 535 \mathrm{~K})$, supporting the idea of different compounds promoted by the excess of cyclopentanone.

Based on these results, 10:1 is chosen as the optimum ratio, obtaining a stable C10 concentration at long TOS, and minimizing the undesired secondary reactions related to FFL oligomers. However, the adsorption of the main products is still too relevant, preventing the use of these conditions for long TOS. This is the starting point of the following experiments, trying to minimize it by changing other reaction conditions.

\subsection{Effect of the reaction $\mathrm{pH}$ on the catalyst performance.}

The $\mathrm{pH}$ of the reaction medium affects the surface charge of the solid and, therein, tunes the adsorption mechanism and strength of reactants and products. In order to set an appropriate $\mathrm{pH}$ range for this study, the isoelectric point of $\mathrm{Mg}-\mathrm{Zr}$ was determined, obtaining a value of 7.8. It is expected that the neutral catalytic surface obtained at this point hinders adsorption processes involved in the catalytic deactivation since electrostatic forces are minimized. 
Three different solutions were prepared with a final $\mathrm{pH}=7.8$ (prepared with potassium bicarbonate) and 9 (with potassium hydroxide, since this $\mathrm{pH}$ cannot be reached with the previous salt), in order to test this effect in the interval close to the isoelectric point. Activity results are compared in Figure 5, showing also the previous results obtained at 4.75 (original $\mathrm{pH}$ of the ethanol/water solution) to make easier the comparison. In all the cases, CPO:FFL initial ratio of 10:1 is defined, according to main conclusions of the previous section. As in the previous section, the detailed evolution of all the profiles is included in the supplementary information (Fig. S8). It must be pointed out that furfural acetylation is not observed in any of these cases, as it could be expected since this reaction is promoted by acidity and reactions are carried out in basic conditions [37].

Reaction at $\mathrm{pH}=9$ is discarded, based on the lowest $\mathrm{C} 15$ yield observed even at low TOS as well as the worst carbon balances obtained at long TOS ( $80 \%$, whereas more than $92 \%$ were obtained in the other cases). It is assumed that the introduction of $\mathrm{OH}^{-}$in the medium strongly modifies the basic sites, increasing the strongest ones. According to the literature, these sites promote oligomerizations $[14,39,40]$, which explains the behavior of all the involved compounds. No significant improvement is observed in the reaction at $\mathrm{pH} 7$, in comparison to the original reaction at $\mathrm{pH} 4.75$, with a slight increase in the carbon balance closure (most likely because of the weakening of adsorption effects), and small increase in the C15 yield. At these conditions, an almost complete deactivation is observed, as in the case of $\mathrm{pH} 4.75$. 

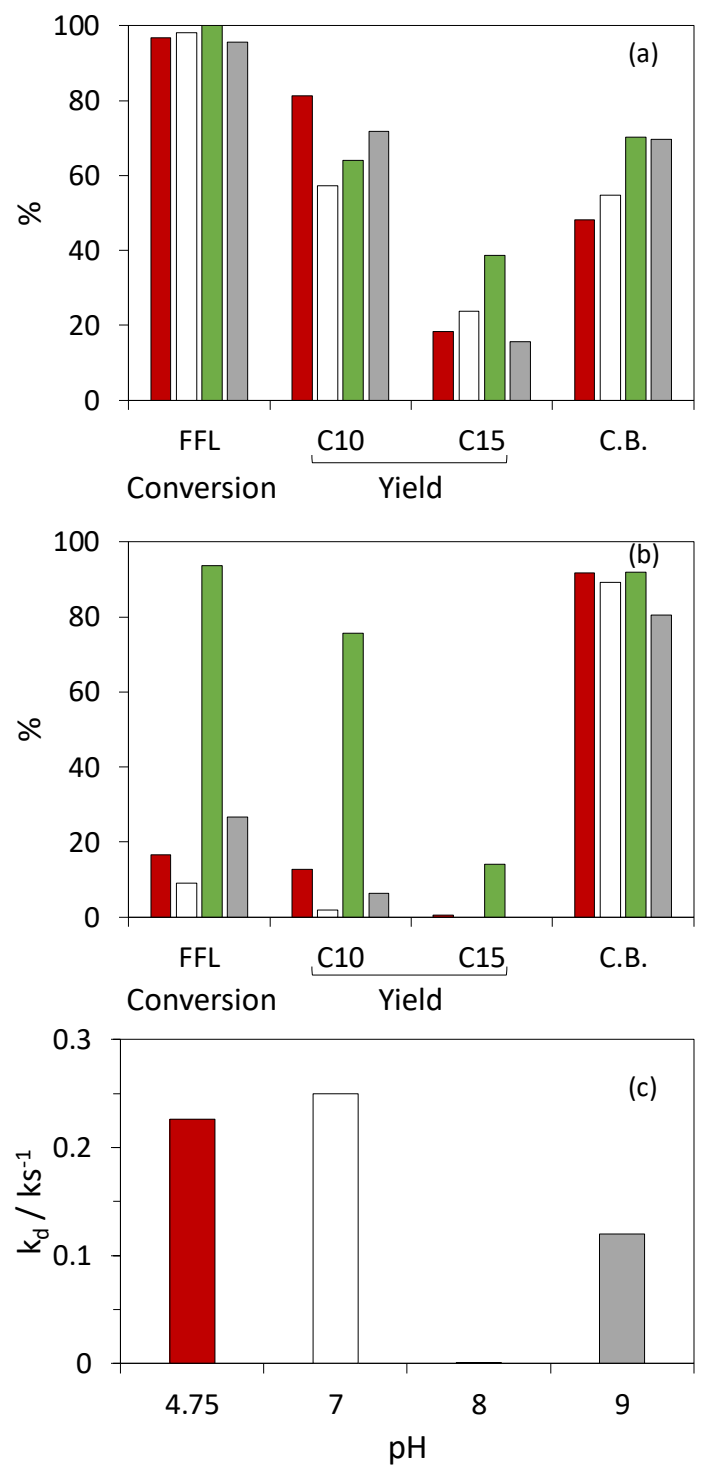

Figure 5: Main data obtained in the CPO and FFL condensation (10:1) at $323 \mathrm{~K}$ as function of the $\mathrm{pH}$. Values related to: (a) $t=0 ;$ (b) $t=8$ h; (c) deactivation kinetic constant. Data correspond to $\mathrm{pH} 4.75$ (red); 7 (white); 8 (green); 9 (grey).

A highlighting different behavior is observed when working at $\mathrm{pH} 8$ (value close to the experimental isoelectric point), reaching almost $100 \%$ of FFL conversion during the $8 \mathrm{~h}$. The relevance of homogeneous mechanism at these conditions was also evaluated by substituting the $\mathrm{Mg}-\mathrm{Zr}$ catalyst by glass-balls, obtaining less than $4 \mathrm{mmol} \cdot \mathrm{L}^{-1}$ of $\mathrm{C} 10$ after $8 \mathrm{~h}$. Consequently, homogeneous contribution has a negligible relevance, being responsible of less than $8 \%$ of FFL 
conversion obtained at the final point at these conditions. To conclude, heterogeneous mechanism prevails over the homogeneous one, and results can be directly compared in terms of Mg-Zr activity. This high activity allows obtaining initial yields of C15 close to $40 \%$ at initial TOS, with a progressive decrease in its trend to a final $14 \%$ after $8 \mathrm{~h}$, whereas a complete disappearance is observed for the other reaction pHs. This result is congruent with a higher solubility of this adduct at $\mathrm{pH} 8$, reducing its deposition and, consequently, the catalytic deactivation. However, the most significant change corresponds to $\mathrm{C} 10$ profile, with a constant $70-72 \%$ yield (value that corresponds to $50 \mathrm{mmol} \cdot \mathrm{L}^{-1}$ ). This flat trend strongly contrasts to the decreasing one obtained in the other cases, with an almost null concentration of this product at the larger times on stream.

Concerning the carbon balance, closures higher than $90 \%$ are obtained in these three cases, with relevant decreases at the initial times (up to $73 \%$ ) tentatively explained by adsorption processes. This adsorption only corresponds to $\mathrm{CPO}$, since there is a good concordance between FFL conversion and reaction products.

To sum up, $\mathrm{pH}$ is related not only to stability but also to the intrinsic catalytic activity of the catalyst: the high conversion obtained at $\mathrm{pH} 8$ does not imply the total absence of deactivation, as it is observed in the $\mathrm{C} 15$ evolution. According to these results, reactions with lower catalytic $(0.25,0.1 \mathrm{~g})$ amount were carried out, with the aim to reduce the initial $100 \%$ conversion (not conclusive results, possible masking of deactivation), allowing a deep analysis of the prevalence of these two effects: activity and stability. These results are compared with those previously obtained with $0.5 \mathrm{~g}$ in Figure 6 , being the time evolution shown in the supplementary information (Figure S9). 


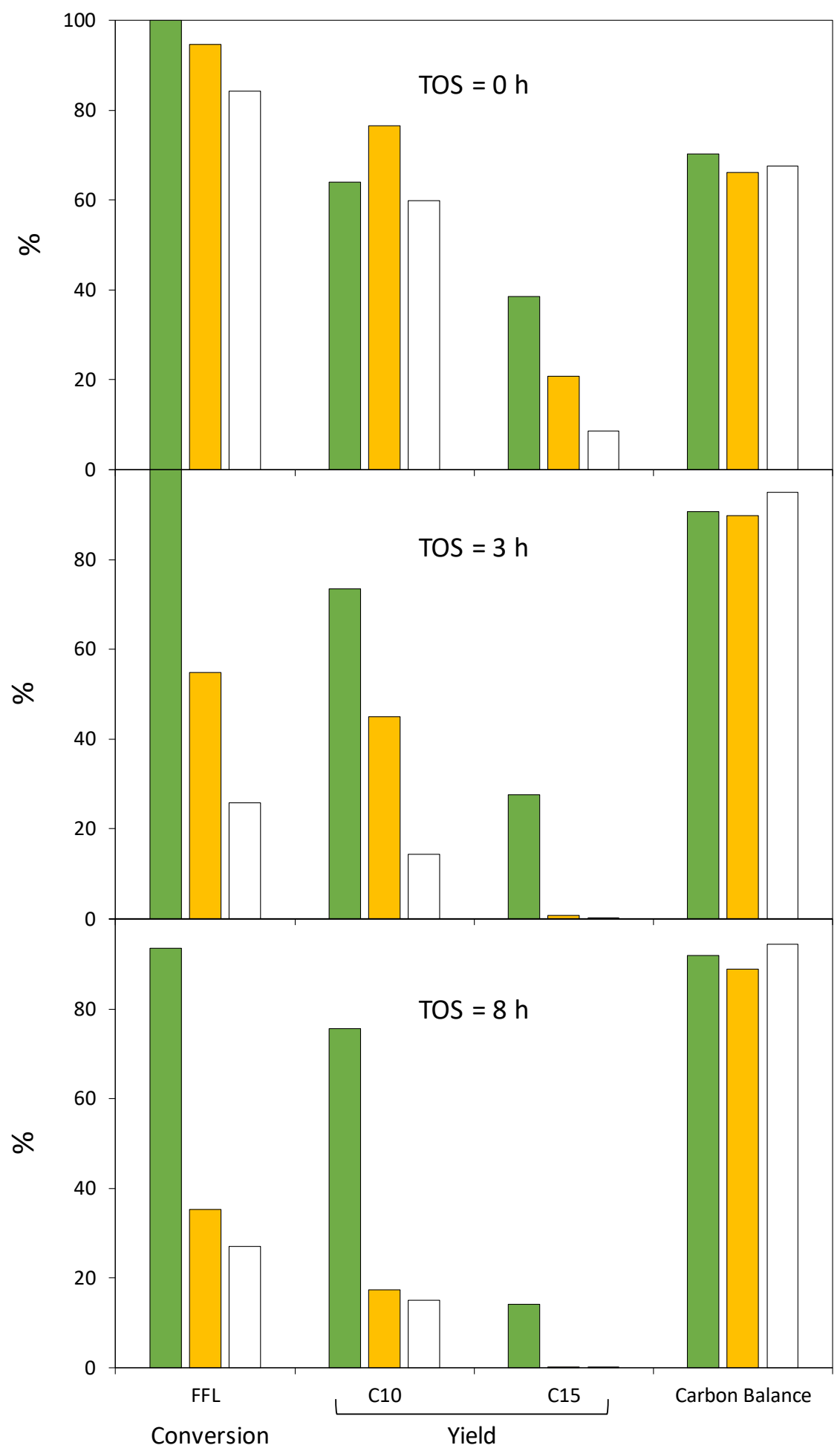

Figure 6: Main data obtained in the CPO and FFL condensation $(10: 1, \mathrm{pH}=8)$ at $323 \mathrm{~K}$ as function of the catalytic loading. Results corresponding to initial time, 3 and $8 \mathrm{~h}$ TOS. Colors: (green) $0.5 \mathrm{~g}$; (yellow) 0.25 g; (white) $0.1 \mathrm{~g}$. 
Conversion significantly falls as catalyst concentration decreases, observing the partial deactivation previously supposed. Nevertheless, stable values are obtained at long TOS, with final furfural conversion of $27 \%$. This value is reached after only 30 min on stream when working with $0.1 \mathrm{~g}$, whereas it seems to be the asymptotic value of the evolution obtained with $0.25 \mathrm{~g}$. In the same way, a stable $15 \%$ of final C10 yield is observed. This value corresponds to a concentration of $9 \mathrm{mmol} \cdot \mathrm{L}^{-1}$, and it is not congruent with $\mathrm{FFL}$ conversion ( $27 \%$, equivalent to a consumption of $\left.14 \mathrm{mmol} \cdot \mathrm{L}^{-1}\right)$, suggesting that $\mathrm{pH}=8$ also promotes the reaction to obtain mainly $\mathrm{C} 15$. At this point, it must be mentioned that only relevant signals of $\mathrm{C} 10$ were obtained at $\mathrm{pH}=4.75$. At $\mathrm{pH} 8$, the high activity produces local oversaturation, enhancing the precipitation and producing the progressive deactivation. However, this deactivation is not as strong as in previous cases, being these results congruent with a weaker interaction product-surface at these conditions. Thus, some active sites are clean to continue with the reaction for longer times. Other deactivation causes, such as leaching (ICP analyses), are discarded, obtaining only $0.7 \mathrm{ppm}$ of $\mathrm{Mg}$ in the final liquid phase ( $86 \%$ of reduction respect to the original $50 \mathrm{ppm}$ observed at $\mathrm{pH} 4.75)$.

In order to check the proposed hypotheses, a reusability test was carried out. As it was expected, no conversion was obtained with the direct reuse of spent catalysts, whereas very good results are achieved if the catalyst is recovered by filtration and air-treated after the first cycle of $8 \mathrm{~h}$ (12 h at $473 \mathrm{~K}$ ). Main results are compared in Table 1, with a negligible decrease in conversion and yields. These results are congruent to a deactivation due to $\mathrm{C} 15$ deposits; since FFL oligomers would be stable under $600 \mathrm{~K}$ (as shown in Figure S4).

However, this discussion does not explain the marked activity increase observed at $\mathrm{pH}=8$, since catalyst stability decreases again at $\mathrm{pH}=9$. Thus, a relevant role of the salt used for reaching this $\mathrm{pH}$, and the consequence side effects produced by the $\mathrm{KHCO}_{3}$. 
Table 1. Summary of the final conversions and yields obtained in the stability test of $\mathrm{Mg}-\mathrm{Zr}$ for FFL-CPO aldol condensation in a continuous plug flow reactor. Operation conditions: $4.6 \mathrm{~h}^{-1}$, $323 \mathrm{~K}, 2: 1 \mathrm{EtOH} /$ Water and $\mathrm{pH} 8$.

\begin{tabular}{c|c|c|c}
\cline { 2 - 4 } & FFL Conv. (\%) & $\boldsymbol{\varphi}$ C10 (\%) & $\boldsymbol{\varphi}$ C15 (\%) \\
\hline First Cycle & 93.7 & 75.7 & 14.2 \\
\hline Second Cycle & 89.3 & 76.7 & 12.5 \\
\hline
\end{tabular}

\subsection{Interaction between bicarbonate and catalytic surface}

The role of both ions of the salt (potassium and bicarbonate) is studied comparing reactions at $\mathrm{pH}=8$ using different salts for increasing the basicity of the medium, combining different cations and anions. Figure 7 shows the main results obtained using $\mathrm{KOH}, \mathrm{NaOH}, \mathrm{KHCO}_{3}$ and $\mathrm{NaHCO}_{3}$. The global profiles are included in the Supplementary Information (Figure S10). Best results are obtained with the initial $\mathrm{KHCO}_{3}$, both in terms of activity and selectivity. However, interesting conclusions can be achieved by analyzing the different behaviors.

A clear role of the cation is observed at initial TOS, with common full conversion of FFL when using $\mathrm{K}^{+}$, but a lower value when using the $\mathrm{Na}^{+}(\approx 70 \%)$, despite the anion. Although the presence of $\mathrm{KOH}$ leads to high FFL conversion, this conversion does not lead to high $\mathrm{C} 10$ or $\mathrm{C} 15$ productivities, suggesting an increase of the extent of side reactions. At this point, several authors have stated that hydroxyl anions promote base-catalyzed unselective reactions $[14,39,40]$. The situation is completely different when the reaction is performed in presence of $\mathrm{KHCO}_{3}$. In this case, the trend observed for FFL matches to an increase of the productivity of both reaction products. The buffer effect of bicarbonates could explain its better behavior due to its capacity for protons scavenging. This analysis at low TOS, then, discards the use of hydroxyls and suggests a relevant role of $\mathrm{K}^{+}$enhancing the catalytic activity. 

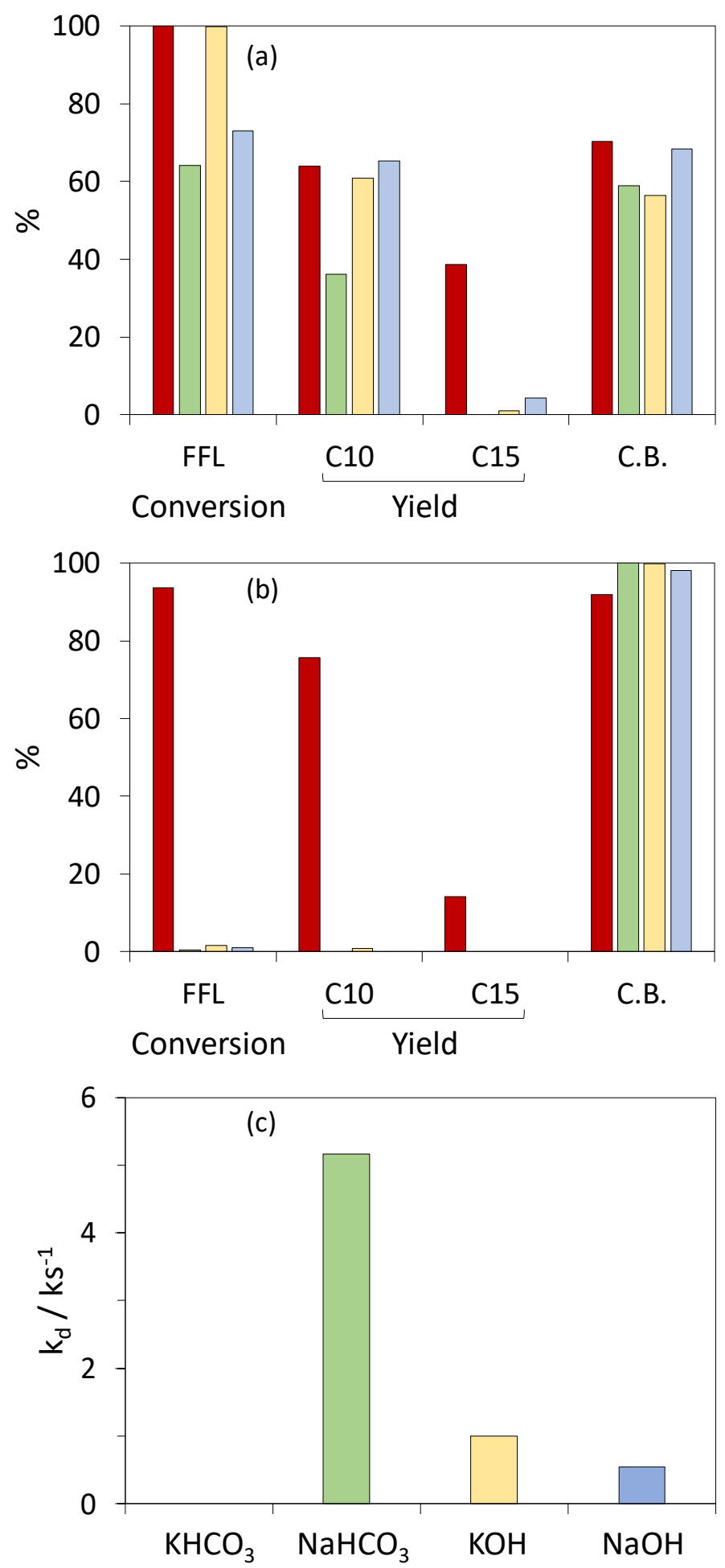

Figure 7: Main data obtained in the $\mathrm{CPO}$ and $\mathrm{FFL}$ condensation $(10: 1, \mathrm{pH}=8,0.5 \mathrm{~g}$ catalyst) at $323 \mathrm{~K}$ as function of salt used to keep $\mathrm{pH}$ at 8 . Comparison between (a) initial TOS; and (b) t=8h. Results correspond to: (red) $\mathrm{KHCO}_{3}$; (green) $\mathrm{NaHCO}_{3}$; (yellow) $\mathrm{KOH}$; (blue) $\mathrm{NaOH}$. Graph (c) indicates the deactivation kinetic constant values obtained 
This increase in the activity must be related to the number of basic sites that are active under reaction conditions. As the strength of these sites is also dependent on the exchanged cations and the framework $\mathrm{Mg}$ atoms, ICP analyses of different liquid phases when using both bicarbonates were done. Results obtained analyzing the amount of $\mathrm{Mg}, \mathrm{Na}$ and $\mathrm{K}$ in the liquid phase are summarized in Figure 8. Relevant differences are observed, mainly after only $2 \mathrm{~h}$ on stream. The Mg leaching is totally prevented in presence of $\mathrm{KHCO}_{3}$, whereas $10.2 \mathrm{ppm}$ of this metal is detected in the liquid phase when working with $\mathrm{NaHCO}_{3}$. Concerning the $\mathrm{Zr}$, this reduction is less remarkable, which is congruent to a very low leaching trend despite the conditions (from 7 to 4 and $3.5 \mathrm{ppb}$, in absence of any salt and with $\mathrm{NaHCO}_{3}$ and $\mathrm{KHCO}_{3}$, respectively). These results are not plotted in Figure 8 because of the scale.
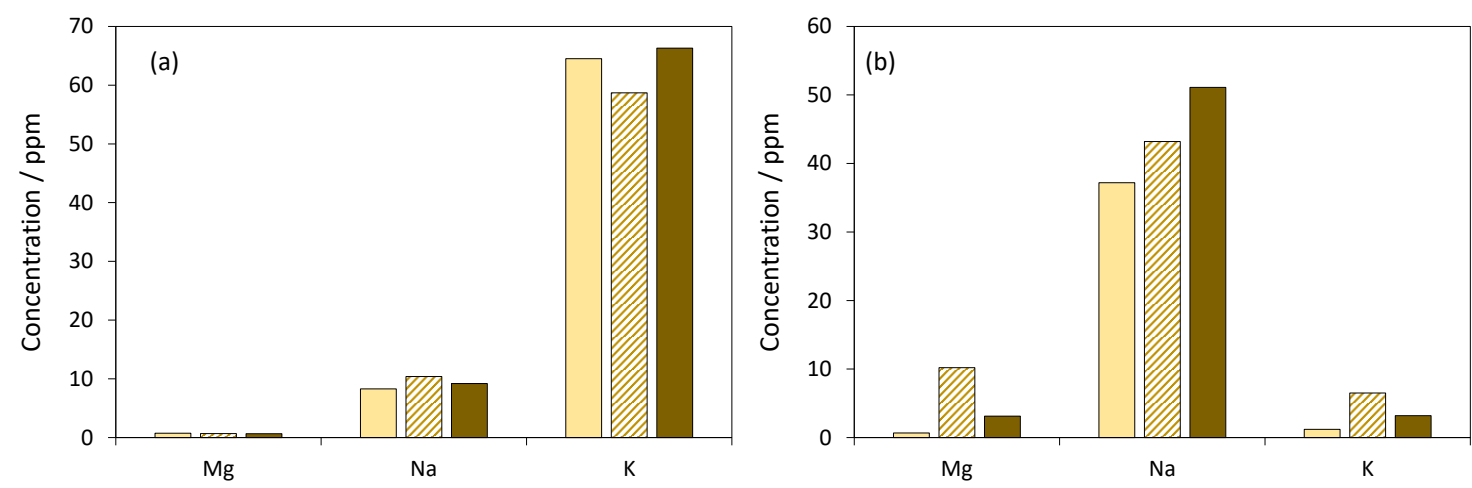

Figure 8. Evolution of metal dissolved in the liquid phase as function of the reaction time.

Results corresponding to using (a) $\mathrm{KHCO}_{3}$; and (b) $\mathrm{NaHCO}_{3}$ as salts to reach the reaction $\mathrm{pH}$.

Symbols: (light organge) TOS $=0 \mathrm{~h}$; (gratted bars) TOS $=2 \mathrm{~h}$; (dark brown) TOS $=8 \mathrm{~h}$.

Even more relevant than the $\mathrm{Mg}$ is the evolution of the alkaline cations $\left(\mathrm{Na}^{+}\right.$and $\left.\mathrm{K}^{+}\right)$ concentration in the liquid phase. It must be pointed out that some traces of sodium are always detected in the catalytic leaching, since $\mathrm{NaOH}$ is used for increasing the $\mathrm{pH}$ during the preparation procedure $[14,17]$ and some cations remain in the structure after the washing step. The $\mathrm{Na}^{+}$signal detected in the feed stream, as well as after 2 and $8 \mathrm{~h}$ on stream, keeps constant in $10 \mathrm{ppm}$ when working with $\mathrm{KHCO}_{3}$, concluding that no changes related to this 
cation are taking place at these conditions. However, when working with $\mathrm{NaHCO}_{3}$, the concentration increases from 37 to 43 and $51 \mathrm{ppm}$ (same samples measured), indicating that some sodium is extracted from the catalytic structure. This effect can modify the mixed oxide surface, affecting to its catalytic activity. However, when reaction occurs in presence of $\mathrm{KHCO}_{3}$, the evolution observed is the opposite, with signals of $\mathrm{K}^{+}$from 65 to 57 and $66 \mathrm{ppm}$, respectively. This decrease during the first two hours on stream suggests that some potassium cations are introduced in the solid structure. This fact can produce a swelling of the structure, increasing the number of active sites exposed to the surface, so available for the reaction, explaining (together with the decrease in the $\mathrm{Mg}$ leaching) the activity increase.

On the other hand, concerning the catalyst stability, all the materials undergo fast deactivation in less than $2.5 \mathrm{~h}$ (see Figure 7), except when working in presence of $\mathrm{KHCO}_{3}$. If the only presence of bicarbonate anions could increase the selectivity, the profiles obtained with $\mathrm{NaHCO}_{3}$ must be more constant, despite the lower initial values. According to this, a synergistic effect of $\mathrm{K}^{+}$and bicarbonate is suggested as the key parameter to enhance the activity and stability in this reaction.

In order to corroborate this discussion, the catalytic preparation procedure was modified to check the effect of including $\mathrm{K}^{+}$in the bulk material or in the surface, analyzing the influence in the catalytic activity. Taking into account the previous results, a Mg-Zr catalyst using $\mathrm{KOH}$ instead of $\mathrm{NaOH}$ was prepared, keeping constant the other steps of the procedure. This catalyst is labelled as $\mathrm{Mg}-\mathrm{Zr}^{*}$. A $\mathrm{CO}_{2}$-TPD analysis of this material corroborates an increase in the global basicity with an enrichment in the medium and strongest sites (desorption temperatures from 373 to $573 \mathrm{~K}$ and higher than $573 \mathrm{~K}$, respectively) and a decrease of almost $30 \%$ in the weakest ones (see Table S2). According to the most accepted mechanism, mediumstrength basic sites are the responsible of the aldol condensation [14], so an increase in the product yields is predictable. 
The activity of this modified catalyst (pink squares) is compared with the previous one (red circles), being the main results shown in Figure 9.
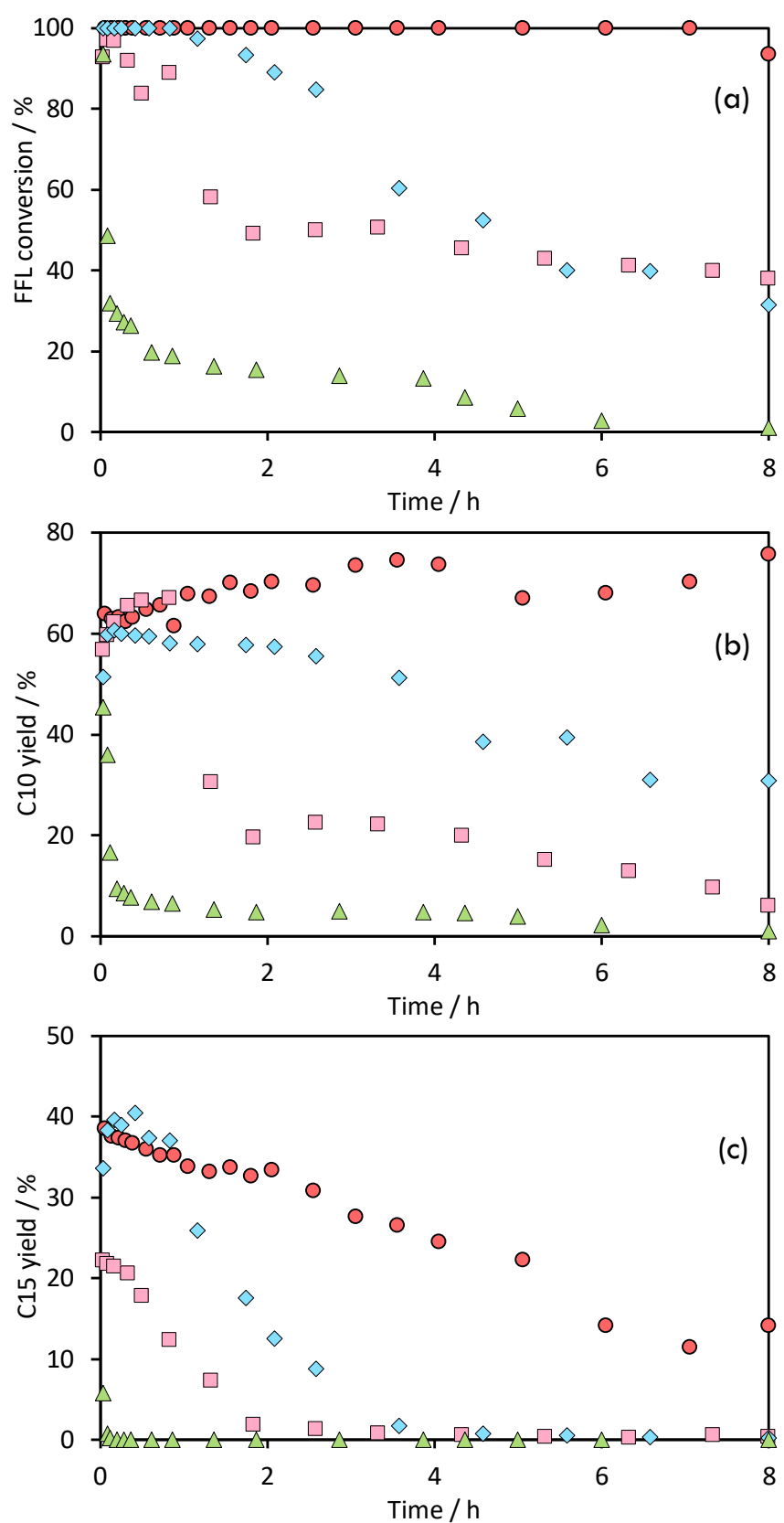

Figure 9: Time evolution of $\mathrm{CPO}$ and FFL condensation at $323 \mathrm{~K}$ as function of the modifications in the preparation method of $\mathrm{Mg}-\mathrm{Zr}$ and the salt used to keep $\mathrm{pH}$ at 8. Data corresponds to (a) FFL conversion; (b) $\mathrm{C} 10$ yield; and (c) $\mathrm{C} 15$ yield. Symbols: (๑) $\mathrm{Mg}-\mathrm{Zr}$, using $\mathrm{KHCO}_{3}$ in the feed; ( $\left.\mathbf{\square}\right) \mathrm{Mg}-\mathrm{Zr} *$ using $\mathrm{KHCO}_{3}$; $(\bullet) \mathrm{Mg}-\mathrm{Zr} *$ using $\mathrm{NaHCO}_{3} ;(\boldsymbol{\Delta}) \mathrm{Mg}-\mathrm{Zr}$ washed with $\mathrm{KHCO}_{3}$ and using $\mathrm{NaHCO}_{3}$ for the reaction. 
Both profiles correspond to results at $\mathrm{pH}$ 8, reaching this value using $\mathrm{KHCO}_{3}$. The expected improvement by introducing the $\mathrm{K}$ in the catalytic structure is not reached, observing a clear deactivation after $1 \mathrm{~h}$ TOS, with an almost total disappearance of main products. These low yields disagree with the conversion (higher than $35 \%$ during all the experiment). This fact suggests that side reaction involving FFL oligomerization are taking place, being the possible responsible of the catalytic deactivation. According to these results, the co-presence of both cations, $\mathrm{Na}^{+}$and $\mathrm{K}^{+}$is required to maximize the activity and stability, discarding those combinations that include same cation for preparation and $\mathrm{pH}$ control.

To deep analyze the relevance of each cation; two extra experiments were carried out, being the profiles also shown in Figure 9. The first one involves the catalyst prepared with $\mathrm{KOH}$ ( $\left.\mathrm{Mg}-\mathrm{Zr}^{*}\right)$ using $\mathrm{NaHCO}_{3}$ as salt to increase the $\mathrm{pH}$ (blue diamonds). In this experiment, $\mathrm{K}^{+}$ cations take part of the mixed oxide structure, whereas sodium ones only interact with the surface. The second approach involves a typical $\mathrm{Mg}$ - $\mathrm{Zr}$ mixed oxide (precipitated with $\mathrm{NaOH}$ ) after a washing step with a $\mathrm{KHCO}_{3}$ solution $(1 \mathrm{~h}$ at $323 \mathrm{~K})$, carrying out the reaction at $\mathrm{pH} 8$ with $\mathrm{NaHCO}_{3}$. This experiment corresponds to green triangles in the Figure 9. The aim of this second experiment is to corroborate if the positive effect of $\mathrm{K}^{+}$is due to an ion exchanged on the surface, without any modification in the bulk structure. This last option is discarded, according to the lower initial activity ( 45 and $6 \%$ of $\mathrm{C} 10$ and C15 yields, respectively) and, more relevant, the high deactivation, with complete activity loss after less than $6 \mathrm{~h}$. However, an intermediate behavior is observed with the previous one, with higher activity and selectivity ( $31 \%$ of $\mathrm{C} 10$ yield after $8 \mathrm{~h}$ ) than the ones obtained with same cation for both roles, but lower values than those obtained using $\mathrm{Na}$ for the preparation and $\mathrm{K}$ for the $\mathrm{pH}$ buffering. It can be concluded that the activity and selectivity increases are related to the active exchange between both cations during all the reaction, being promoted by the first combination tested: precipitation with $\mathrm{NaOH}$ and reaction with $\mathrm{KHCO}_{3}$. This fact must be explained according to the 
different interactions between cations and mixed oxide structure, as function of their atomic properties.

The presence of $\mathrm{K}$ ions seems to modify either the strength or the availability of the active basic sites, with an opposite role if potassium is included in the bulk structure or if it is deposed on the catalytic surface. This effect is due to the laminar structure of mixed oxides precursors, similar than the hydrotalcite ones [41]. This structure is only partially damaged during the heat treatment, producing active sites in the corresponding breaking points and discontinuities obtained. At this point, it should be considered that $\mathrm{K}^{+}$has both larger ionic radium (133 pm, vs 95 for $\mathrm{Na}^{+}$and 65 for $\mathrm{Mg}^{2+}$ ) and larger electropositivity than the other two cations (ionization energy of $4.34 \mathrm{eV}$, versus 5.14 and $7.65 \mathrm{eV}$ for $\mathrm{Na}$ and $\mathrm{Mg}$, respectively). The first property will lead to a more irregular surface when $\mathrm{Na}$ is replaced by $\mathrm{K}$, improving the availability of the basic sites. The second one leads to an improved stabilization of the strongest basic sites. In the case of the material prepared using $\mathrm{KOH}$ as precipitating agent, the morphological effect is diminished (there are not exchange of soluble cations of different size), and the stabilization of the strongest basic sites can lead to basic sites more active for side reactions (justifying the poorer selectivities and the higher deactivation). In good agreement with this explanation, any positive effect is observed when the catalyst is prepared using $\mathrm{NaOH}$ as precipitating agent, the catalyst is washed with $\mathrm{KHCO}_{3}$, and the $\mathrm{pH}$ is controlled using sodium salts.

To sum up, a key role of these metals grading the strength of the interaction between the bicarbonate and the catalytic surface is proposed. This interaction modifies the active sites available for the reaction and, in the optimum combination, reduces the permanent adsorption of heavy compounds, with a clear positive effect in the stability. The interaction between the surface and the bicarbonate is too strong when introducing the same cation, resulting into a negative effect in the global activity of this material. 


\section{Conclusions}

CPO-FFL aldol condensation has been studied in a continuous fixed bed reactor, observing a clear deactivation. Reaction experiments as well as spent catalyst characterization allow concluding that this deactivation is due to the deposition of organic compounds derived by C15 oligomerization and side reactions involving FFL.

Reaction conditions were optimized, analyzing the role of initial reactant ratios, $\mathrm{pH}$ and salts involved in adsorption processes, concluding that a high activity increase is obtained with 10:1 CPO:FFL ratio, working at $\mathrm{pH} 8$ reached with $\mathrm{KHCO}_{3}$, with a final global yield $(\mathrm{C} 10+\mathrm{C} 15)$ of $90 \%$ after $8 \mathrm{~h}$ TOS, with $94 \%$ of furfural conversion.

A key role of bicarbonate is observed, suggesting the equilibrium between the $\mathrm{Na}^{+}$and $\mathrm{K}^{+}$on the catalytic surface as the responsible of the stabilization of the active sites needed for obtaining de desired products.

\section{Acknowledgments}

This work has been financed by the Spanish Ministry of Economy and Competitiveness (CTQ2014-52956-C3-1-R and CTQ2017-89443-C3-2-R) and the Regional Government of Asturias (project reference IDI/2018/000116).

\section{Appendix A. Supplementary data}

Detailed information related to mass transfer limitations is included as supplementary information, as well as TG, TPO, and mass spectra of C10 and C15 products. In addition, all the detailed temporal profiles not shown in the main manuscript are also included in the supplementary information, as well as the corresponding discussion, when required. 


\section{References}

[1] J. Pang, J. Sun, M. Zhen, H. Li, W. Wang, T. Zhang, Appl. Catal. B 254 (2019) 510-522. https://doi.org/10.1016/i.apcatb.2019.05.034

[2] R.F. Pérez, O.S.G.P. Soares, A.M.D. de Farias, M.F.R. Pereira, M.A. Fraga, Appl. Catal. B 232 (2018) 101-107. https://doi.org/10.1016/j.apcatb.2018.03.042

[3] C.J. Zimmermann, N.V. Bollar, S.G. Wettstein, Biomass Bioenerg. 118 (2018) 163-171. https://doi.org/10.1016/j.biombioe.2018.08.009

[4] K. Kohli, R. Prajapati, B.K. Sharma, Energies $12 \quad$ (2019) 233. https://doi.org/10.3390/en12020233

[5] J. Cueto, L. Faba, E. Díaz, S. Ordóñez, Appl. Catal. B 201 (2017) 221-231. https://doi.org/10.1016/i.apcatb.2016.08.013

[6] I. Sadaba, M. Ojeda, R. Mariscal, J.L.G. Fierro, M.L. Granados, Appl. Catal. B 101 (2011) 638-648. https://doi.org/1.1016/j.apcatb.2010.11.005

[7] G.W. Huber, J.N. Chheda, C.J. Barrett, J.A. Dumesic, Science 308 (2005) 1446-1450. https://doi.org/10.1126/science.1111166

[8] R.E. O'Neill, L. Vanoye, C. De Bellefon, F. Aiouache, Appl. Catal. B 144 (2014) 46-56. https://doi.org/10.1016/j.apcatb.2013.07.006

[9] O. Kikhtyanin, L. Capek, L. Smolakova, Z. Tisler, D. Kadlec, M. Lhotka, P. Diblikova, D. Kubicka, Ind. Eng. Chem. Res. 56 (2017) 13412-13423. https://doi.org/10.1021/acs.iecr.7b03367

[10] J. Barrett, J. N. Chheda, G. W. Huber, J. A. Dumesic, Appl. Catal. B 66 (2006) 111-118. https://doi.org/10.1016/i.apcatb.2006.03.001

[11] R.M. West, Z.Y. Liu, M. Peter, C.A. Gärtner, J.A. Dumesic, J. Mol. Catal. A 296 (2008) 1827. https://doi.org/10.1016/j.molcata.2008.09.001

[12] L. Hora, O. Kikhtyanin, L. Čapek, O. Bortnovskiy, D. Kubička, Catal. Today 241 (2015) 221230. https://doi.org/10.1016/i.cattod.2014.03.010 
[13] O. Kikhtyanin, Z. Tisler, R. Velvarska, D. Kubicka, Appl. Catal. A 536 (2017) 85-96. https://doi.org/10.1016/j.apcata.2017.02.020

[14] L. Faba, E. Díaz, S. Ordóñez, Appl. Catal. B 113-114 (2012) 201-211. https://doi.org/10.1016/j.apcatb.2011.11.039

[15] R.E. O’Neill, L. Vanoye, C. De Bellefon, F. Aiouache, Appl. Catal. B 144 (2014) 46-56. https://doi.org/10.1016/j.apcatb.2013.07.006

[16] D.T. Ngo, T. Sooknoi, D.E. Resasco, Appl. Catal. B 237 (2018) 835-843. https://doi.org/10.1016/j.apcatb.2018.06.044

[17] O. Kikhtyanin, L. Hora, D. Kubička, Catal. Commun. 58 (2015) 89-92. https://doi.org/10.1016/j.catcom.2014.09.002

[18] J.E. Rekoske, M.A. Barteau, Ind. Eng. Chem. Res. 50 (2011) 41-51. http://doi.org/10.1021/ie100394v

[19] Z.D. Young, S. Hanspal, R.J. Davis, ACS Catal. 6 (2016) 3193-3202. https://doi.org/10.1021/acscatal.6b00264

[20] K.A. Goulas, S. Dery, P. Dietrich, G.R. Johnson, A. Grippo, Y.C.Wang, E. Gross, Catal. Today In press, https://doi.org/10.1016/i.cattod.2018.12.012

[21] J. Cueto, L. Faba, E. Díaz, S. Ordonez, ChemCatChem 9 (2017) 1765-1770. https://doi.org/10.1002/cctc.201601655

[22] G. Zhang, M. Zhu, Q. Zhang, Y. Liu, H. He, Y. Cao, Green Chem. 18 (2016) 2155-2164. https://doi.org/10.1039/c5gc02528a

[23] Y. Yang, Z. Du, Y. Huang, F. Lu, F. Wang, J. Gao, J. Xu Green Chem. 15 (2013) 1932-1940. https://doi.org/10.1039/c3gc37133f

[24] T. Omotoso, L.V. Herrera, T. Vann, N.M. Briggs, L.A. Gómez, L. Barrett, D. Jones, T. Pham, B. Wang, S.P. Crossley, Appl. Catal. B 254 (2019) 491-499. http://doi.org/10.1016/j.apcatb.2019.04.079 
[25] M. Hronec, K. Fulajtarova, I. Vavra, T. Sotak, E. Dobrocka, M. Micusik, Appl. Catal. B 181 (2016) 210-219. http://doi.org/10.1016/i.apcatb.2015.07.046

[26] M. Hronec, K. Fulajtarova, T. Sotak, Appl. Catal. B 154 (2014) 294-300. http://doi.org/10.1016/j.apcatb.2014.02.029

[27] D.T. Ngo, Q.H. Tan, B. Wang, D.E. Resasco, ACS Catal. 9 (2019) 2831-2841. https://doi.org/10.1021/acscatal.8b05103

[28] L. Ao, W. Zhao, Y.S. Guan, D.K. Wang, K.S. Liu, T.T. Guo, X. Fan, X.Y. Wei, RSC Adv. 9 (2019) 3661-3668. https://doi.org/10.1039/c8ra09517e

[29] B. Wozniak, A. Spannenberg, Y.H. Li, S. Hinze, J.G. de Vries, ChemSusChem 11 (2018) 356359. http://doi.org/10.1002/cssc. 201702100

[30] W. Wang, N. Li, G.Y. Li, S.S. Li, W.T. Wang, A.Q. Wang, Y. Cong, X.D. Wang, T. Zhang, ACS Sust. Chem. Eng. 5 (2017) 1812-1817. http://doi.org/10.1021/acssuschemeng.6b02554.

[31] J. Cueto, L. Faba, E. Díaz, S. Ordóñez, J. Chem. Technol. Biotechnol. 93 (2018) 1563-1571. https://doi.org/10.1002/jctb.5522

[32] C. Perego, S. Peratello, Catal. Today 52 (1999) 133-145. https://doi.org/10.1016/X0920$\underline{5861(99) 00071-1}$

[33] J. T. Scanlon, D. E. Willis, J. Chromatogr. Sci. 23 (1985) 333-340. https://doi.org/10.1093/chromsci/23.8.333

[34] J.J. Carberry, AIChE J. 6 (1960) 460-463. https://doi.org/10.1002/aci.690060323

[35] S. E. Stein, R. L. Brown, J. Chem. Inf. Model. 1994, 34, 581-587. https://doi.org/10.1021/ci00019a016

[36] D. Liang, G. Li, Y. Liu, J. Wu, X. Zhang, Catal. Commun. 81 (2016) 33-36. https://doi.org/10.1016/j.ctcom.2016.04.008

[37] J.M. Rubio-Caaballero, S. Saravanamurugan, P. Maireles-Torres, A. Riisager, Catal. Today 234 (204) 233-236. http://doi.org/10.1016/i.cattod.2014.03.004 
[38] M. Wan, D. Liang, L. Wang, X. Zhang, D. Yang, G. Li, J. Catal. 361 (2018) 186-192. http://doi.org/10.1016/i.jcat.2018.02.021

[39] L. Faba, E. Díaz, S. Ordóñez, Catal. Today 164 (2011) 451-456. https://doi.org/10.1016/i.cattod.2010.11.032

[40] Di Cosimo, C. R. Apesteguía, J. Mol. Catal. Chem. 130 (1998) 177-185. https://10.1016/S1381-1169(97)00204-5

[41] W. Yang, W. Kim, P.K.T. Liu, M. Sahimi, T.T. Tsotsis, Chem. Eng. Sci. 57 (2002) 2945-2953. https://doi.org/10.1016/S0009-2509(02)00185-9 
